\title{
ON THE DURRMEYER-TYPE MODIFICATION OF SOME DISCRETE APPROXIMATION OPERATORS
}

\author{
PAULINA PYCH-TABERSKA
}

\begin{abstract}
In [10], for continuous functions $f$ from the domain of certain discrete operators $L_{n}$ the inequalities are proved concerning the modulus of continuity of $L_{n} f$. Here we present analogues of the results obtained for the Durrmeyer-type modification $\widetilde{L}_{n}$ of $L_{n}$. Moreover, we give the estimates of the rate of convergence of $\widetilde{L}_{n} f$ in Hölder-type norms
\end{abstract}

\section{Introduction AND Notation}

Let $I$ be a finite or infinite interval. Consider a sequence $\left(J_{k}\right)_{1}^{\infty}$ of some index sets contained in $Z:=\{0, \pm 1, \pm 2, \ldots\}$, choose real numbers $\xi_{j, k} \in I$ and fix non-negative functions $p_{j, k}$ continuous on $I$. Write, formally,

$$
L_{k} f(x):=\sum_{j=J_{k}} f\left(\xi_{j, k}\right) p_{j, k}(x) \quad(x \in I, k \in N:=\{1,2, \ldots\})
$$

for univariate (complex-valued) functions $f$ defined on $I$. If for $f_{0}(x) \equiv 1$ on $I$ the values $L_{k} f_{0}(x)(x \in I, k \in N)$ are finite, then $L_{k} f$ are well-defined for every function $f$ bounded on $I$. Under appropriate additional assumptions, operators (1) are meaningful also for some locally bounded functions $f$ on infinite intervals $I$. The fundamental approximation properties of operators (1) in the space $C(I)$ of all continuous functions on $I$ can be deduced, for example, via the general Bohman-Korovkin theorems ([5], Sect. 2.2).

Recently, several authors have investigated relations between the smoothness properties of the functions $f$ and $L_{k} f$ ([1], [10], [15]). For example, taking an arbitrary function $f \in C(I) \cap \operatorname{Dom}\left(L_{n}\right), n \in N$, Kratz and Stadtmüller [10] obtained the following result. Let

$$
\sum_{j \in J_{k}} p_{j, k}(x) \leq c_{1} \quad \text { for all } x \in I, \quad k \in N,
$$

1991 Mathematics Subject Classification. 41A17, 41A25, 26A15. 
and let the sum of the above series be independent of $x$; if, moreover,

$$
p_{j, k}^{\prime} \in C(\stackrel{\circ}{I}), \quad \sum_{j \in J_{k}}\left|\left(\xi_{j . k}-x\right) p_{j, k}^{\prime}(x)\right| \leq c_{1}^{\prime} \quad \text { for all } \quad x \in \stackrel{\circ}{I}, \quad k \in N,
$$

where $c_{1}, c_{1}^{\prime}$ are positive constants and $\stackrel{\circ}{I}$ denotes the interior of $I$, then the ordinary moduli of continuity of $f$ and $L_{n} f$ satisfy the inequality

$$
\omega\left(L_{n} f ; \delta\right) \leq 2\left(c_{1}+c_{1}^{\prime}\right) \omega(f ; \delta) \quad(\delta \geq 0) .
$$

They proved an analogous inequality for the suitable weighted moduli of continuity of $f$ and $L_{n} f$ when $I$ is an infinite interval and $f$ has the modulus $|f|$ of polynomial growth at infinity. In [12] their result is extended to functions $f$ having $|f|$ of a stronger growth than the polynomial one. [12] also presents some applications of the above-mentioned inequalities in problems of approximation of continuous functions $f$ by $L_{n} f$ in some Hölder-type norms.

Suppose that for every $j \in J_{k}$ and every $k \in N$ the integral $\int_{I} p_{j, k}(t) d t$ coincides with a positive number, say, $1 / q_{j, k}$. Denote by $\widetilde{L}_{k}$ the operators given by

$$
\widetilde{L}_{k} f(x) \equiv \widetilde{L}_{k}(f)(x):=\sum_{j \in J_{k}} q_{j, k} p_{j, k}(X) \int_{I} f(t) p_{j, k}(t) d t \quad(x \in I, \quad k \in N)
$$

for these measurable (complex-valued) functions $f$ for which the right-hand side of (3) is meaningful. This modification of the classical Bernstein polynomials was first introduced by J.I. Durrmeyer (see [4]). The approximation properties of these polynomials were investigated, for example, in [4], [7], [2]. Some results on the approximation of functions by the Durrmeyertype modification of the Szász-Mirakyan operators, Baskakov operators or Meyer-König and Zeller operators can be found, for example, in [8], [9], [13], [14], [16].

In this paper we derive Kratz and Stadtmüller type inequalities involving ordinary or weighted moduli of continuity of the functions $f$ and $\widetilde{L}_{n} f$ on $I$. Using these inequalities, we obtain estimates of the degree of approximation of $f$ by $\widetilde{L}_{n} f$ in some Hölder-type norms. Theorems $1-3$ show that the smoothness properties of $\widetilde{L}_{n} f$ are slightly different from those of $L_{n} f$.

We adopt the following notation. Given any non-negative function $w$ defined on $I$ and any $x, y \in I$, we write $\check{w}(x, y):=\min \{w(x), w(y)\}$.

For an arbitrary function $f$ defined on $I$ we introduce the quantities

$$
\begin{gathered}
\|f\|_{w}:=\sup \{|f(x)| w(x): \quad x \in I\}, \\
\Omega_{w}(f ; \delta):=\sup \{|f(x)-f(y) \| \check{w}(x, y): x, y \in I,| x-y \mid \leq \delta\} \quad(\delta \geq 0) .
\end{gathered}
$$


If $f$ is continuous on $I$ and $\|f\|_{w}<\infty$, we say that $f \in C_{w}(I)$. The quantity $\Omega_{w}(f ; \delta)$ is called the weighted modulus of continuity of $f$ on $I$. In case $w(x)=1$ for all $x \in I, \Omega_{w}(f ; \delta)$ becomes $\omega(f ; \delta)$ and the symbol $\|f\|$ is used instead of $\|f\|_{w}$. If the weight $w$ is nondecreasing [nonincreasing] on $I$, then

$\Omega_{w}(f ; \delta):=\sup \{|f(x)-f(y)| w(x)\} \quad\left[\Omega_{w}(f ; \delta):=\sup \{|f(x)-f(y)| w(y)\}\right]$,

where the supremum is taken over all $x, y \in I$ such that $0<y-x \leq \delta$.

We denote by $W$ the set of all continuous functions $w$ on $I$ with values not greater than 1 , which are positive in the interior of $I$ and satisfy the inequality $\check{w}(x, y) \leq w(t)$ for any three points $x, t, y \in I$ such that $x \leq t \leq y$ (obviously, this inequality holds if, for example, $w$ is nondecreasing, nonincreasing or concave on $I$ ). When $I$ is an infinite interval, we indtroduce, in addition, the set $\Lambda$ of all positive functions $\eta$ belonging to $W$ such that $\eta(x) \rightarrow 0$ as $|x| \rightarrow 0$.

Given two weights $w, \eta \in W$, we define a more general modulus of continuity of $f$ on $I$ by

$$
\Omega_{w, \eta}(f ; \delta):=\sup \{|f(x)-f(y)| \check{w}(x, y) \check{\eta}(x, y): x, y \in I,|x-y| \leq \delta\} .
$$

It reduces to $\Omega_{w}(f ; \delta)$ if $\eta \equiv 1$ on $I$, and to $\Omega_{\eta}(f ; \delta)$ if $w \equiv 1$ on $I$. Taking into account that the positive function $\varphi$ is nondecreasing on the interval $(0,1]$ and has values not greater that 1 , we put

$$
\begin{gathered}
\|f\|_{w, \eta}^{(\varphi)}:=\|f\|_{w \eta}+ \\
+\sup \left\{\frac{|f(x)-f(y)| \check{w}(x, y) \check{\eta}(x, y)}{\varphi(|x-y|)}: x, y \in I, \quad<|x-y| \leq 1\right\} .
\end{gathered}
$$

If this quantity is finite, we call it the Hölder-type norm of $f$ on $I$. Under the assumption $f \in C_{\eta}(I),\|f\|_{w, \eta}^{(\varphi)}<\infty$ if and only if there exists a positive constant $K$ such that $\Omega_{w, \eta}(f ; \delta) \leq K \varphi(\delta)$ for every $\delta \in(0,1]$. We write $\|f\|_{w}^{(\varphi)}$ for $\|f\|_{w, \eta}^{(\varphi)}$ if $\eta \equiv 1$ on $I$, and $\|f\|_{\eta}^{(\varphi)}$ if $w \equiv 1$ on $I$.

Throughout this paper the symbols $c_{\nu}(\nu=1,2, \ldots)$ will mean some positive constants depending only on a given sequence $\left(L_{k}\right)_{1}^{\infty}$ and eventually on the considered weights $w, \eta, \rho$. The integer part of the real number will be denoted by $[a]$.

\section{Smoothness Properties}

Let $\widetilde{L}_{k}, k \in N$, be the operators defined by (3) such that $\widetilde{L}_{k} f_{0}(x)$ are finite at every $x \in I$. Put

$$
r_{k}(x):=\sum_{j \in J_{k}} p_{j, k}(x)-1 \quad(x \in I, \quad k \in N)
$$


and make the standing assumption that all functions $p_{j, k}\left(j \in J_{k}, k \in N\right)$ are absolutely continuous on every compact interval contained in $I$. Consider measurable functions $f$ locally bounded on $I$ and belonging to $\operatorname{Dom}\left(\widetilde{L}_{n}\right)$ for some $n \in N$. Write, as in Section $1, \stackrel{\circ}{I}=\operatorname{Int} I$.

Theorem 1. Suppose that condition (2) is satisfied and

$$
\sum_{j \in J_{k}} q_{j, k}\left|p_{j, k}^{\prime}(x)\right| \int_{I}|t-x| p_{j, k}(t) d t \leq \frac{c_{2}}{w(x)}
$$

for $x \in \stackrel{\circ}{I}$ and all $k \in N, w$ being a function of the class $W$. Then

$$
\Omega_{w}\left(\widetilde{L}_{n} f ; \delta\right) \leq c_{3} \omega(f ; \delta)+\|f\|_{w} \omega\left(r_{n} ; \delta\right) \quad(\delta \geq 0),
$$

where $c_{3}=2\left(c_{1}\|w\|+c_{2}\right)$.

Proof. Let $x, y \in I 0<y-x \leq \delta$ and let $x_{0}:=(x+y) / 2$. Clearly,

$$
\begin{gathered}
\widetilde{L}_{n} f(x)-\widetilde{L}_{n} f(y)=\sum_{j \in J_{n}} q_{j, n}\left(p_{j, n}(x)-p_{j, n}(y)\right) \int_{I}\left(f(t)-f\left(x_{0}\right)\right) p_{j, n}(t) d t+ \\
+f\left(x_{0}\right)\left(r_{n}(x)-r_{n}(y)\right) .
\end{gathered}
$$

Taking into account (2) and the well-known inequality $\left|f(t)-f\left(x_{0}\right)\right| \leq$ $\left(1+\left[\left|t-x_{0}\right| \delta^{-1}\right]\right) \omega(f ; \delta)$, we obtain $\left|\widetilde{L}_{n} f(x)-\widetilde{L}_{n} f(y)\right| \leq\left(2 c_{1}+A_{n}(x, y)\right) \times$ $\omega(f ; \delta)+\left|f\left(x_{0}\right)\right| \omega\left(r_{n} ; \delta\right)$, where

$$
\begin{aligned}
A_{n}(x, y) & :=\sum_{j \in J_{n}} q_{j, n}\left|p_{j, n}(x)-p_{j, n}(y)\right| \delta^{-1} \int_{I \backslash I_{\delta}}\left|t-x_{0}\right| p_{j, n}(t) d t \leq \\
\leq & \delta^{-1} \int_{x}^{y}\left(\sum_{j \in J_{n}} q_{j, n}\left|p_{j, n}^{\prime}(s)\right| \int_{I \backslash I_{\delta}}\left|t-x_{0}\right| p_{j, n}(t) d t\right) d s
\end{aligned}
$$

and $I_{\delta}:=I \cap\left(x_{0}-\delta, x_{0}+\delta\right)$. If $x<s<y$ and $\left|t-x_{0}\right| \geq y-x$, then $\left|t-x_{0}\right| \leq 2|t-s|$. Hence, applying (4), we get

$$
A_{n}(x, y):=2 \delta^{-1} \int_{x}^{y}\left(\sum_{j \in J_{n}} q_{j, n}\left|p_{j, n}^{\prime}(s)\right| \int_{I}|t-s| p_{j, n}(t) d t\right) d s \leq 2 c_{2} \delta^{-1} \int_{x}^{y} \frac{1}{w(s)} d s,
$$

and inequality (5) follows.

The result of Theorem 1 is interesting if $\omega(f ; \delta)<\infty$. This holds, for example, for functions $f \in C(I)$ on the compact interval $I$. If $I$ is an infinite interval, the assumption $\omega(f ; \delta)<\infty$ implies the restriction $f(x)=O(|x|)$ as $|x| \rightarrow \infty$. So, in this case, it is convenient to use the weighted modulus of continuity $\Omega_{\eta}(f ; \delta)$ with some $\eta \in \Lambda$. If $f \in C_{\eta}(I)$, then this modulus 
is a nondecreasing function of $\delta$ on the interval $[0, \infty)$. It is easy to verify that, for every $\delta>0$ and for all $x, y \in I$ there holds the inequality

$$
|f(x)-f(y)| \check{\eta}(X, y) \leq\left(1+\left[\delta^{-1}|x-y|\right]\right) \Omega_{\eta}(f ; \delta) .
$$

Moreover, in case $\rho \in \Lambda$ and $\rho(x) / \eta(x) \rightarrow 0$ as $|x| \rightarrow \infty$ we have $\Omega_{\rho}(f ; \delta) \rightarrow$ 0 as $\delta \rightarrow 0+$, whenever $f \in C_{\eta}(I)$ is uniformly continuous on each finite interval contained in $I$.

Note that under the assumptions $\eta \in \Lambda, f \in C_{\eta}(I)$ and $\widetilde{L}_{k}(1 / \eta)(x)<\infty$ we have $\left|L_{k} f(x)\right|<\infty$. If, moreover, $\rho \in \Lambda$ and

$$
\widetilde{L}_{k}\left(\frac{1}{\eta}\right)(x) \leq \frac{c_{4}}{\rho(x)} \text { for all } x \in I \text { and } k \in N
$$

then $\left\|\widetilde{L}_{k} f\right\|_{\rho}<\infty$.

In the next two theorems it is assumed that $I$ is an infinite interval.

Theorem 2. Let condition (2) be satisfied. Suppose, moreover, that there exist functions $w \in W, \rho, \eta \in \Lambda, \rho \leq \eta$ such that (4), (8) and

$$
\begin{aligned}
& \sum_{j \in J_{k}} q_{j, k}\left|p_{j, k}^{\prime}(x)\right| \int_{I} \frac{|t-x|}{\eta(t)} p_{j, k}(t) d t \leq \\
& \leq \frac{c_{5}}{w(x) \rho(x)} \text { for a.e. } x \in \stackrel{\circ}{I} \text { and } k \in N
\end{aligned}
$$

hold. Then

$$
\Omega_{w, \rho}\left(\widetilde{L}_{n} f ; \delta\right) \leq c_{6} \Omega_{\eta}(f ; \delta)+\|f\|_{w \rho} \omega\left(r_{n} ; \delta\right) \quad(\delta \geq 0),
$$

where $c_{6}=2\left(\left(c_{1}+c_{4}\right)\|w\|+c_{2}+c_{5}\right)$.

Proof. Consider $x, y \in I$ such that $0<y-x \leq \delta$. Retain the symbol $x_{0}$ used in the proof of Theorem 1 and start with identity (6). In view of (7), $\left|\widetilde{L}_{n} f(x)-\widetilde{L}_{n} f(y)\right| \leq B_{n}(x, y) \Omega_{\eta}(f ; \delta)+\left|f\left(x_{0}\right)\right|\left|r_{n}(x)-r_{n}(y)\right|$, where

$$
B_{n}(x, y):=\sum_{j \in J_{n}} q_{j, n}\left|p_{j, n}(x)-p_{j, n}(y)\right| \int_{I}\left(1+\left[\delta^{-1}\left|t-x_{0}\right|\right]\right) \frac{1}{\check{\eta}\left(t, x_{0}\right)} p_{j, n}(t) d t .
$$

Observing that for every $t \in I$

$$
\frac{\check{\rho}(x, y)}{\check{\eta}\left(t, x_{0}\right)} \leq 1+\frac{\check{\rho}(x, y)}{\eta(t)}
$$


and applying (2), we obtain

$$
\begin{gathered}
B_{n}(x, y) \check{\rho}(x, y) \leq 2 c_{1}+\sum_{j \in J_{n}} q_{j, n}\left|p_{j, n}(x)-p_{j, n}(y)\right| \int_{I} \frac{\check{\rho}(x, y)}{\eta(t)} p_{j, n}(t) d t+ \\
\quad+\delta^{-1} \sum_{j \in J_{n}} q_{j, n} \int_{x}^{y}\left|p_{j, n}^{\prime}(s)\right| d s \int_{I-I_{\delta}}\left(1+\frac{\check{\rho}(x, y)}{\eta(t)}\right)\left|t-x_{0}\right| p_{j, n}(t) d t .
\end{gathered}
$$

Further, the inequality $\left|t-x_{0}\right| \leq 2|t-s|\left(t \in I \backslash I_{\delta}, x<s<y\right)$ and assumptions (4), (8), (9) lead to

$$
B_{n}(x, y) \check{\rho}(x, y) \leq 2\left(c_{1}+c_{4}\right)+2 \delta^{-1} \int_{x}^{y} \frac{c_{2}+c_{5}}{w(s)} d s .
$$

The desired estimate is now evident.

For functions $f$ for which $|f|$ is of the polynomial growth at infinity our result can be stated as follows.

Theorem 3. Let conditions (2), (4) be satisfied and let $\eta(x)=(1+|x|)^{-\sigma}$ $x \in I \sigma>0$. Suppose that inequality (9) in which $\rho=\eta$ holds. Then

$$
\Omega_{w, \eta}\left(\widetilde{L}_{n} f ; \delta\right) \leq c_{7} \Omega_{\eta}(f ; \delta)+\|f\|_{w \eta} \omega\left(r_{n} ; \delta\right) \quad(\delta \geq 0)
$$

where $c_{7}=2\left(c_{1}+2 \cdot 3^{\sigma} c_{1}+c_{2}+2 c_{5}\right)$.

Proof. To see this it is enough to make a slight modification in the evaluation of the term $B_{n}(x, y)$ occurring in the proof of Theorem 2. Namely, let us divide the interval $I$ into two sets $I_{n}$ and $I \backslash I_{h}$, where $I_{h}:=I \cap\left(x_{0}-h, x_{0}+h\right)$, $h=y-x$. If $t \in I_{h}$, then $\left[\delta^{-1}\left|t-x_{0}\right|\right]=0$ and

$$
\frac{\check{\eta}(x, y)}{\eta(t)} \leq 3^{\sigma} \check{\eta}(x, y)\left(\frac{1}{\eta(x)}+\frac{1}{\eta(y)}\right) \leq 2 \cdot 3^{\sigma} .
$$

This inequality, (11) and (2) imply

$$
\begin{gathered}
B_{n}(x, y) \check{\eta}(x, y) \leq 2\left(1+2 \cdot 3^{\sigma}\right) c_{1}+ \\
+\sum_{j \in J_{n}} q_{j, n}\left|p_{j, n}^{\prime}(s) d s\right| \int_{I \backslash I_{h}}\left(\frac{\left|t-x_{0}\right|}{\delta}+\frac{\check{\eta}(x, y)}{\eta(t)}\left(1+\frac{\left|t-x_{0}\right|}{y-x}\right)\right) p_{j, n}(t) d t .
\end{gathered}
$$


Observing that $\left|t-x_{0}\right| \leq 2|t-s|,\left|t-x_{0}\right| \leq y-x$ whenever $t \in I \backslash I_{h}$, $x<s<y$, we obtain, on account of (4) and (9) (with $\rho=\eta$ ),

$$
\begin{gathered}
B_{n}(x, y) \check{\eta}(x, y) \leq 2\left(1+2 \cdot 3^{\sigma}\right) c_{1}+ \\
+\frac{2}{\delta} \int_{x}^{y} \frac{c_{2}}{w(s)} d s+4 \frac{\check{\eta}(x, y)}{y-x} \int_{x}^{y}\left(\sum_{j \in J_{n}} q_{j, n}\left|p_{j, n}^{\prime}(s)\right| \int_{I} \frac{|t-s|}{\eta(t)} p_{j, n}(t) d t\right) d s \leq \\
\leq 2\left(1+2 \cdot 3^{\sigma}\right) c_{1}+\frac{2}{y-x} \int_{x}^{y} \frac{c_{2}+c_{5}}{w(s)} d s .
\end{gathered}
$$

Thus

$$
B_{n}(x, y) \check{w}(x, y) \check{\eta}(x, y) \leq 2\left(1+2 \cdot 3^{\sigma}\right) c_{1}\|w\|+2 c_{2}+4 c_{5} .
$$

Remark 1. For many known operators the functions $r_{k}(x) \equiv 0$ on $I$, the quantities $\mu_{2, k}(x):=\sum_{j \in J_{k}}\left(\xi_{j, k}-x\right)^{2} p_{j, k}(x)$ are finite at every $x \in I$ and positive in $\stackrel{\circ}{I}$; moreover,

$$
p_{j, k}^{\prime}(x) \mu_{2, k}(x)=p_{j, k}(x)\left(\xi_{j, k}-x\right)
$$

for every $x \in \stackrel{\circ}{I}$ and every $k \in N$. In view of identity (12) and the CauchySchwartz inequality the left-hand side of (4) can be estimated from above by $\left(\widetilde{\mu}_{2, k}(x) / \mu_{2, k}(x)\right)^{1 / 2}$, where $\widetilde{\mu}_{2, k}(x):=\sum_{j \in J_{k}} q_{j, k}\left|p_{j, k}(x)\right| \int_{I}(t-x)^{2} p_{j, k}(t) d t$. Therefore, in this case, assumption (4) can be replaced by

$$
\frac{\widetilde{\mu}_{2, k}(x)}{\mu_{2, k}(x)} \leq \frac{c_{2}^{2}}{w^{2}(x)} \text { for all } x \in \stackrel{\circ}{I}, k \in N
$$

Analogously, the left-hand side of (9) can be estimated by

$$
\frac{1}{\mu_{2, k}(x)}\left(\widetilde{\mu}_{2, k}(x) \sum_{j \in J_{k}} q_{j, k}\left(\xi_{j, k}-x\right)^{2} p_{j, k}(x) \int_{I} \frac{p_{j, k}(t)}{\eta^{2}(t)} d t\right)^{1 / 2} .
$$

Hence, if

$$
\frac{1}{\mu_{2, k}(x)} \sum_{j \in J_{k}} q_{j, k} p_{j, k}(x)\left(\xi_{j, k}-x\right)^{2} \int_{I} \frac{p_{j, k}(t)}{\eta^{2}(t)} d t \leq \frac{c_{8}^{2}}{\rho^{2}(x)}
$$

for all $x \in \stackrel{\circ}{I}, k \in N$, then (9) holds with $c_{5}=c_{2} \cdot c_{8}$. 
Remark 2. Let $w \in W, \eta \in \Lambda$. Define the weighted modulus $\Phi_{w}(f ; \delta)$ and $\Phi_{w, \eta}(f ; \delta)$ as in Section 1, replacing $\check{w}(x, y)$ by

$$
\bar{w}(x, y):= \begin{cases}0 & \text { if } w(x)=0 \quad \text { or } w(y)=0, \\ \left(\frac{1}{w(x)}+\frac{1}{w(y)}\right)^{-1} & \text { otherwise, }\end{cases}
$$

and $\check{\eta}(x, y)$ by $\bar{\eta}(x, y)$, respectively. Since $\bar{w}(x, y) \leq \check{w}(x, y)$ for every pair of points $x, y \in I$, Theorem 1 remains valid for $\Phi_{w}\left(\widetilde{L}_{n} f ; \delta\right)$. Further, in this case, inequality (7) becomes $|f(x)-f(y)| \bar{\eta}(x, y) \leq 2\left(1+\left[\delta^{-1} \mid x-\right.\right.$ $y[]) \Phi_{\eta}(f ; \delta)$. Consequently, under the assumptions of Theorem 2, the modulus $\Phi_{w, \rho}\left(\widetilde{L}_{n} f ; \delta\right)$ and $\Phi_{\eta}(f ; \delta)$ satisfy inequality (10) with the constant $2 c_{6}$ instead of $c_{6}$.

Note that, for the weight $\eta(x)=(1+|x|)^{-\sigma}$ with the parameter $\sigma>0$, the modulus $\Phi_{\eta}(f ; \delta)$ is equivalent to the one introduced in [10], p. 331 (see also [12]).

\section{Approximation Properties}

Considering still the functions $f$ as in Section 2 we first estimate the ordinary weighted norm of the difference $\widetilde{L}_{n} f-f$.

Theorem 4. Let condition (2) be satisfied and let

$$
\begin{aligned}
& \rho(x) \widetilde{L}_{k}\left(\frac{1}{\eta^{2}}\right)(x) \leq \frac{c_{9}}{\eta(x)} \quad \text { for all } \quad x \in I, \quad k \in N, \\
& \rho(x) \widetilde{\mu}_{2, k}(x) \leq c_{10} \eta(x) \delta_{k}^{2} \quad \text { for all } \quad x \in I, \quad k \in N,
\end{aligned}
$$

where $\left(\delta_{k}\right)_{1}^{\infty}$ is a sequence of positive numbers, $\eta$ is a positive function on $I$ and $\rho$ is a non-negative one such that $\rho \leq \eta$. Then

$$
\left\|\widetilde{L}_{n} f-f\right\|_{\rho} \leq c_{11} \Omega_{\eta}\left(f ; \delta_{n}\right)+\|f\|_{\rho}\left\|r_{n}\right\|,
$$

where $c_{11}=c_{1}+\left(c_{1} c_{9}\right)^{1 / 2}+\left(c_{9} c_{10}\right)^{1 / 2}+c_{10}$.

Proof. Start with the obvious identity

$$
\widetilde{L}_{n} f(x)-f(x)=\sum_{j \in J_{n}} q_{j, n} p_{j, n}(x) \int_{I}(f(t)-f(x)) p_{j, n}(t) d t+f(x) r_{n}(x)
$$

and take a positive number $\delta$. In view of $(7)$ and the inequality $(\check{\eta}(x, t))^{-1} \leq$ $(\eta(x))^{-1}+(\eta(t))^{-1}$ we have $\left|\widetilde{L}_{n} f(x)-f(x)\right| \leq \gamma_{n}(x) \Omega_{\eta}(f ; \delta)+|f(x)| \cdot\left\|r_{n}\right\|$, where

$$
\gamma_{n}(x):=\sum_{j \in J_{n}} q_{j, n} p_{j, n}(x) \int_{I}\left(1+\left[\delta^{-1}|t-x|\right]\right)\left(\frac{1}{\eta(x)}+\frac{1}{\eta(t)}\right) p_{j, n}(t) d t .
$$


Further, by (2), (15) and (16) and the Cauchy-Schwartz inequality we obtain

$$
\begin{gathered}
\gamma_{n}(x) \rho(x) \leq c_{1}+\widetilde{L}_{n}\left(\frac{1}{\eta}\right)(x) \rho(x)+\delta^{-2} \frac{\rho(x)}{\eta(x)} \widetilde{\mu}_{2, n}(x)+ \\
+\rho(x) \delta^{-1} \sum_{j \in J_{n}} q_{j, n} p_{j, n}(x) \int_{I} \frac{|t-x|}{\eta(t)} p_{j, n}(t) d t \leq \\
\leq c_{1}+\left(c_{1} \widetilde{L}_{n}\left(\frac{1}{\eta^{2}}\right)(x)\right)^{1 / 2} \rho(x)+c_{10} \delta^{-2} \delta_{n}^{2}+ \\
+\rho(x) \delta^{-1}\left(\widetilde{\mu}_{2, n}(x)\right)^{1 / 2}\left(\widetilde{L}_{n}\left(\frac{1}{\eta^{2}}\right)(x)\right)^{1 / 2} \leq \\
\leq c_{1}+\left(c_{1} c_{9}\right)^{1 / 2}+c_{10} \delta^{-2} \delta_{n}^{2}+\left(c_{9} c_{10}\right)^{1 / 2} \delta^{-1} \delta_{n} .
\end{gathered}
$$

Choosing $\delta=\delta_{n}$, we get (17) at once.

Remark 3. In the case when $\eta(x)=1$ for all $x \in I$, the constant $c_{11}$ in (17) is equal to $c_{1}+c_{10}$. If we use the modulus $\Phi_{\eta}(f ; \delta)$ (defined in Remark 2 ) instead of $\Omega_{\eta}(f ; \delta)$, the constant $c_{11}$ should be multiplied by 2 .

Passing to approximation in the Hölder-type norm we note that, for an arbitrary $\nu_{n} \in(0,1]$

$$
\begin{gathered}
\left\|\widetilde{L}_{n} f-f\right\|_{w, \eta}^{(\varphi)} \leq\left(1+\frac{2}{\varphi\left(\nu_{n}\right)}\right)\left\|\widetilde{L}_{n} f-f\right\|_{w \eta}+ \\
+\sup \left\{\frac{1}{\varphi(\delta)}\left(\Omega_{w, \eta}\left(\widetilde{L}_{n} f ; \delta\right)+\Omega_{w, \eta}(f ; \delta)\right): 0<\delta \leq \nu_{n}\right\}
\end{gathered}
$$

(see, for example, [11], [12]). This inequality, Theorem 4 and the estimates obtained in Section 2 allow us to state a few standard results. We will formulate only one of them. Namely, combining inequality (18) with Theorems 1 and 2 gives

Theorem 5. Let conditions (2), (4) be satisfied and let $\left(\delta_{k}\right)_{1}^{\infty}$ be a sequence of numbers from $(0,1]$ for which $(16)$ holds with $\rho=w$ and $\eta \equiv 1$ on I. Then

$$
\left\|\widetilde{L}_{n} f-f\right\|_{w}^{(\varphi)} \leq c_{12} \sup \left\{\frac{\omega(f ; \delta)}{\varphi(\delta)}: 0<\delta \leq \delta_{n}\right\}+\|f\|_{w} \Delta_{n}^{(\varphi)},
$$

where $c_{12}=3 c_{1}+2 c_{2}+3 c_{10}+\left(1+2 c_{1}\right)\|w\|$ and

$$
\Delta_{n}^{(\varphi)}=3\left\|r_{n}\right\| / \varphi\left(\delta_{n}\right)+\sup \left\{\omega\left(r_{n} ; \delta\right) / \varphi(\delta): 0<\delta \leq \delta_{n}\right\} .
$$

Remark 4. Clearly, if the assumptions of Theorems $1--5$ hold for positive integers $k$ belonging to a certain subset $N_{1}$ of $N$, then the corresponding assertions remain valid only if $n \in N_{1}$. 


\section{EXAmples}

1) The Bernstein polynomials $B_{k} f \equiv L_{k} f$ are defined by (1) with $\xi_{j, k}=$ $j / k, p_{j, k}=\left(\begin{array}{c}k \\ j\end{array}\right) x^{j}(1-x)^{k-j}, I=[0,1], J_{k}=\{0,1,2, \ldots, k\}$. The corresponding Bernstein-Durrmeyer polynomials $\widetilde{L}_{k} f \equiv \widetilde{L}_{k} f$ are of the form (3) in which $q_{j, k}=k+1$ for all $j \in J_{k}, k \in N$. In this case $r_{k}(x)=0$ for all $x \in I$, the constant $c_{1}$ in (2) equals $1, \mu_{2, k}(x)=x(1-x) / k$ and equality (12) is true. Since $\widetilde{\mu}_{2, k}(x)=\frac{2 x(1-x)(k-3)+2}{(k+2)(k+3)}(x \in I, \quad k \in N)$ (see [4]), we easily state that condition (13) is satisfied with $c_{2}=1, w(x)=(x(1-x))^{1 / 2}$. Hence, in view of Theorem 1 (and Remark 1), for every $f \in C(I)$ and every $n \in N$, $\Omega_{w}\left(\widetilde{B}_{n} f ; \delta\right) \leq 3 \omega(f ; \delta)(\delta \geq 0)$ Further, $\widetilde{\mu}_{2, k}(x) \leq \frac{1}{2 k}$ for all $x \in I, k \in N$ (see [4], p. 327). Therefore (16) holds with $\rho(x)=\eta(x)=1$ for all $x \in I$, $\delta_{k}=k^{-1 / 2}$ and $c_{10}=1 / 2$. Thus Theorem 4 gives $\left\|\widetilde{B}_{n} f-f\right\| \leq \frac{3}{2} \omega\left(f ; n^{-1 / 2}\right)$ for all $n \in N$ (cf. [4], Theorem II.2). Also, Theorem 5 applies with $w(x)=(x(1-x))^{1 / 2}, \delta_{n}=n^{-1 / 2}, c_{12}=8$ and $\Delta_{n}^{(\varphi)}=0$.

2) The Meier-König and Zeller operators $M_{k} \equiv L_{k}$ are defined by $\xi_{j, k}=$ $j /(j+k), p_{j, k}(x)=\left(\begin{array}{c}k+j-1 \\ j\end{array}\right) x^{j}(1-x)^{k}, x \in I=[0,1), j \in J_{n}=N_{0}$, $N_{0}:=\{0,1, \ldots\}$. Their Durrmeyer modification $\widetilde{M} \equiv \widetilde{L}_{k}$ are of the form (3) in which $q_{j, k}=(k+j)(k+j+1) / k$. Condition (2) holds with $c_{1}=1$. Since

$$
p_{j, k}^{\prime}(x) \frac{x(1-x)^{2}}{k}=p_{j, k+1}(x)\left(\frac{j}{k+j}-x\right)^{2} \quad(0<x<1),
$$

the left-hand side of (4) can be estimated from above by

$$
\begin{aligned}
& \frac{k}{x(1-x)^{2}}\left(\left\{\sum_{j=0}^{\infty}\left(\frac{j}{k+j}-x\right)^{2} p_{j, k+1}(x)\right\} \times\right. \\
& \left.\times\left\{\sum_{j=0}^{\infty} q_{j, k} p_{j, k+1}(x) \int_{0}^{1}(t-x)^{2} p_{j, k}(t) d t\right\}\right)^{1 / 2}
\end{aligned}
$$

for all $x \in(0,1), k \in N$. If $k \geq 3$, the expression in the first curly brackets is not greater than $2 x(1-x)^{2} / k$ (see [3]); straightforward calculation shows that the expression in the second ones does not exceed $7(1-x)^{2} / k$. Thus, for the functions $f \in C(I) \cap \operatorname{Dom}\left(\widetilde{M}_{n}\right)$ and $\widetilde{M}_{n} f(n \geq 3)$, inequality (5) applies with $c_{3}=10, w(x)=x^{1 / 2}$ and $r_{n}(x)=0$ for all $x \in I$.

3) The Baskakov-Durrmeyer operators $\widetilde{U}_{k, c} \equiv \widetilde{L}_{k}$ (with a parameter $c \in N_{0}$ ) are defined by (3) in which $I=[0, \infty), J_{k}=N_{0}, p_{j, k}(x)=$ $(-1)^{j} x^{j} \psi_{k, c}^{(j)}(x) / j !, \psi_{k, c}(x)=e^{-k x}$ if $c=0$, and $\psi_{k, c}(x)=(1+c x)^{-k / c}$ if $c \geq 1, q_{j, k}=k-c$ for $k>c$ (see [9]). Now $r_{k}(x)=0$ for all $x \in I, k \in N$, 
$c_{1}=1, \mu_{2, k}(x)=x(1+c x) / k$ for all $x \in I, k>c$ and condition (12) holds with $\xi_{j, k}=j / k$. Further,

$$
\widetilde{\mu}_{2, k}=\frac{2 x(1+c x)(k+3 c)+2}{(k-2 c)(k-3 c)} \text { for } \quad x \in I, \quad k>3 c .
$$

Hence Theorem 1 (via Remarks 1, 4) applies for $n>3 c$, with $w(x)=$ $(x /(1+x))^{1 / 2}, c_{3}=2\left(1+c_{2}\right), c_{2}=(2(1+3 c)(1+6 c) /(1+c))^{1 / 2}$.

4) The Szász-Mirakyan-Durrmeyer operators $\widetilde{S}_{k}$ are the special case of operators $\widetilde{U}_{k, c}$ defined in 3), with $c=0$. From 3 ) we know that, for these operators, conditions (2) and (13) hold with $c_{1}=1, c_{2}=2^{1 / 2}$ and $w(x)=$ $(x /(1+x))^{1 / 2}$. Consider $f \in C_{\eta}(I)$ with the weight $\eta(x)=(1+x)^{-\sigma}$ where $\sigma \in N$. It is easy to see that, for $k \geq 2 \sigma$,

$$
\begin{gathered}
\int_{0}^{\infty} \frac{1}{\eta^{2}(t)} p_{j, k}(t) d t=\frac{k^{j}}{j !} \int_{0}^{\infty}(1+t)^{2 \sigma} t^{j} e^{-k t} d t \leq 2^{2 \sigma-1}\left(\frac{1}{k}+\frac{k^{j}}{j !} \int_{0}^{\infty} t^{2 \sigma+j} e^{-k t} d t\right)= \\
=2^{2 \sigma-1} \frac{1}{k}\left(1+\frac{(2 \sigma+j) !}{j !} k^{-2 \sigma}\right) \leq 2^{2 \sigma-1} \frac{1}{k}\left(1+\left(\frac{j}{k}+1\right)^{2 \sigma}\right) .
\end{gathered}
$$

Consequently, the left-hand side of (14) is not greater than

$$
\begin{gathered}
\frac{2^{2 \sigma-1}}{\mu_{2, k}(x)} \sum_{j=0}^{\infty}\left(\frac{j}{k}-x\right)^{2} p_{j, k}(x)\left(1+2^{2 \sigma-1}\left((1+x)^{2 \sigma}+\left(\frac{j}{k}-x\right)^{2 \sigma}\right)\right)= \\
=2^{2 \sigma-1}\left(1+2^{2 \sigma-1}(1+x)^{2 \sigma}\right)+\frac{4^{2 \sigma-1}}{\mu_{2, k}(x)} \sum_{j=0}^{\infty}\left(\frac{j}{k}-x\right)^{2 \sigma+2} p_{j, k}(x) \leq \\
\leq c_{13}(1+x)^{2 \sigma}
\end{gathered}
$$

(see [10], p. 334). Applying Theorem 3 (together with Remarks 1, 4), we get the estimate

$$
\Omega_{w, \eta}\left(\widetilde{S}_{n} f ; \delta\right) \leq c_{14} \Omega_{\eta}(f ; \delta) \quad(\delta \geq 0, \quad n \geq 2 \sigma) .
$$

Since $\widetilde{\mu}_{2, k}(x) \leq 2(1+x) / k$, conditions (15) and (16) are satisfied with $\rho(x)=(1+x)^{-\sigma-1}$ and $\delta_{k}=k^{-1 / 2}$. Consequently, Theorem 4 gives

$$
\left\|\widetilde{S}_{n} f-f\right\|_{\rho} \leq c_{15} \Omega_{\eta}\left(f ; n^{-1 / 2}\right) \text { for all } n \in N .
$$

Combining this result and (19) with the general inequality (18), we easily verify that, for $n \geq 2 \sigma$,

$$
\left\|\widetilde{S}_{n} f-f\right\|_{w, \rho}^{(\varphi)} \leq c_{16} \sup \left\{\frac{1}{\varphi(\delta)} \Omega_{\eta}(f ; \delta): 0<\delta \leq n^{-1 / 2}\right\} .
$$


5) The generalized Favard operators $F_{k} \equiv L_{k}$ are deefined by (1) with $\xi_{j, k}=j / k, J_{k}=Z, I=(-\infty, \infty)$ and

$$
p_{j, k}(x) \equiv p_{j, k}(\gamma ; x)=\left(\sqrt{2 \pi} k \gamma_{k}\right)^{-1} \exp \left(-\frac{1}{2} \gamma_{k}^{-2}\left(\frac{j}{k}-x\right)^{2}\right),
$$

$\gamma=\left(\gamma_{k}\right)_{1}^{\infty}$ being a positive null sequence satisfying

$$
k^{2} \gamma_{k}^{2} \geq \frac{1}{2} \pi^{-2} \log k \text { for } k \geq 2, \quad \gamma_{1}^{2} \geq \frac{1}{2} \pi^{-2} \log 2
$$

(see [6]). Denote by $\widetilde{F}_{k}$ their Durrmeyer modification of form (3) in which $q_{j, k}=k$ for all $j \in Z$ and $k \in N$. As is known ([6], [12]), for all $x \in I$ and $k \in N$,

$$
\left|r_{k}(x)\right| \equiv\left|r_{k}(\gamma ; x)\right|=\left|\sum_{j=-\infty}^{\infty} p_{j, k}(\gamma ; x)-1\right| \leq 2 \text { or }\left|r_{k}(\gamma ; x)\right| \leq 7 \pi \gamma_{k} .
$$

$\mu_{2, k}(x) \equiv \mu_{2, k}(\gamma ; x) \leq 51 \gamma_{k}^{2}$; moreover, $\omega\left(r_{k}(\gamma ; x) \mid \leq 16 \pi \delta\right.$ for every $\delta \geq 0$ (see [10], p. 336). It is easy to see that

$$
\widetilde{\mu}_{2, k}(x) \equiv \widetilde{\mu}_{2, k}(\gamma ; x)=\mu_{2, k}(\gamma ; x)+\gamma_{k}^{2}\left(1+r_{k}(\gamma ; x)\right) \leq 54 \gamma_{k}^{2} .
$$

Observing that

$$
p_{j, k}^{\prime}(\gamma ; x)=\gamma_{k}^{-2}\left(\frac{j}{k}-x\right) p_{j, k}(\gamma ; x)
$$

and applying the Cauchy-Schwartz inequality, we estimate the left-hand side of (4) by

$$
\begin{gathered}
k \gamma_{k}^{-2} \sum_{j=-\infty}^{\infty}\left|\frac{j}{k}-x\right| p_{j, k}(\gamma ; x) \int_{-\infty}^{\infty}|t-x| p_{j, k}(\gamma ; t) d t \leq \\
\leq \gamma_{k}^{-2}\left(\mu_{2, k}(\gamma ; x)\right)^{1 / 2}\left(\widetilde{\mu}_{2, k}(\gamma ; x)\right)^{1 / 2},
\end{gathered}
$$

i.e., $w(x)=1$ for all real $x$ and $c_{2}=52,5$. Thus Theorem 1 yields the estimate

$$
\omega\left(\widetilde{F}_{n} f ; \delta\right) \leq 111 \omega(f ; \delta)+16 \pi \delta\|f\| \quad(\delta \geq 0)
$$

for every $n \in N$ and every $f \in C(I)$. Clearly, this inequality is interesting if $f \in C(I)$ is bounded on $I$.

Consider now $f \in C_{\eta}(I)$ where $\eta(x)=\exp \left(-\sigma x^{2}\right) \sigma>0$. If $\sigma \gamma_{k}^{2} \geq 3 / 32$, then

$$
\begin{aligned}
& \exp \left(\sigma x^{2}\right) \exp \left(-\frac{1}{2} \gamma_{k}^{-2}\left(\frac{j}{k}-x\right)^{2}\right) \exp \left(-\frac{1}{2} \gamma_{k}^{-2}\left(\frac{j}{k}-t\right)^{2}\right) \leq \\
& \leq \exp \left(4 \sigma x^{2}\right) \exp \left(-\frac{1}{8} \gamma_{k}^{-2}\left(\frac{j}{k}-x\right)^{2}\right) \exp \left(-\frac{1}{8} \gamma_{k}^{-2}\left(\frac{j}{k}-t\right)^{2}\right)
\end{aligned}
$$


whence

$$
\widetilde{F}_{k}(1 / \eta)(x) \leq 2\left(1+r_{k}(2 \gamma ; x)\right) \exp \left(4 \sigma x^{2}\right)
$$

Analogously, one can show that the left-hand side of (9) is not greater than

$$
\left.2 \gamma_{k}^{-2} \mu_{2, k}(\gamma ; x)\right)^{1 / 2}\left(\widetilde{\mu}_{2, k}(2 \gamma ; x)\right)^{1 / 2} \exp \left(4 \sigma x^{2}\right)
$$

provided that $\sigma \gamma_{k}^{2} \leq 3 / 64$. Further (see [12]),

$$
r_{k}(2 \gamma ; x) \leq 2 / 15, \quad \mu_{2, k}(2 \gamma ; x) \leq 23 \gamma_{k}^{2}
$$

and

$$
\tilde{\mu}_{2, k}(2 \gamma ; x)=\mu_{2, k}(2 \gamma ; x)+\left(2 \gamma_{k}\right)^{2}\left(1+r_{k}(2 \gamma ; x)\right) \leq \frac{413}{15} \gamma_{k}^{2} .
$$

Thus Theorem 2 applies with $w(x) \equiv 1, \rho(x)=\exp \left(-4 \sigma x^{2}\right), c_{4}=68 / 15$, $c_{5}=75$ (i.e. $c_{6}=271$ ) and $n$ such that $\sigma \gamma_{n}^{2} \leq 3 / 64$. In the same way one can show that Theorem 4 is true with $\rho(x)=\rho_{1}(x):=\exp \left(-7 \sigma x^{2}\right)$, $\delta_{n}=\gamma_{n}, \sigma \gamma_{n}^{2} \leq 3 / 64$ and a positive absolute constant $c_{11}$. From these results the estimate of $\left\|\widetilde{F}_{n} f-f\right\|_{\rho_{1}}^{(\varphi)}$ follows at once via inequality (18).

\section{REFERENCES}

1. G.A. Anastassiou, C. Cottin and H.H. Gonska, Global smoothness of approximating functions. Analysis 11(1991), 43-57.

2. G. Aniol and P. Pych-Taberska, On the rate of convergence of the Durrmeyer polynomials. Ann. Soc. Math. Pol., ser. I, Commentat. 30(1990), 9-17.

3. M. Becker and R.J. Nessel, A global approximation theorem for Meyer-König and Zeller operators. Math. Z. 160(1978), 195-206.

4. M.M. Derriennic, Sur l'approximation de fonctions intégrables sur $[0,1]$ par des polynômes de Bernstein modifies. J. Approximation Theory 31(1991), 325-343.

5. R.A. DeVore, The approximation of continuous functions by positive linear operators. Lecture Notes in Mathematics, v. 293, Springer-Verlag, 1972.

6. W. Gawronski and U. Stadtmüller, Approximation of continuous functions by generalized Favard operators. J. Approximation Theory 34(1982), 384-396.

7. S. Guo, On the rate of convergence of the Durrmeyer operator for functions of bounded variation. J. Approximation Theory 51(1987), 183192.

8. S. Guo, Degree of approximation to functions of bounded variation by certain operators. Approximation Theory Appl. 4(1988), 9-18.

9. M. Heilmann, Direct and converse results for operators of BaskakovDurrmeyer type. Approximation Theory Appl. 5(1989), 105-127. 
10. W. Kratz and U. Stadtmüller, On the uniform modulus of continuity of certain discrete approximation operators. J. Approximation Theory 54(1988), 326-337.

11. L. Leidler, A. Meir and V. Totik, On approximation of continuous functions in Lipschitz norms. Acta Math. Hung. 45(1985), No. 3-4, 441-443.

12. P. Pych-Taberska, Properties of some discrete approximation operators in weighted function spaces. Ann. Soc. Math. Pol., Ser. I, Commentat. 33(1993), to appear.

13. A. Sahai and G. Prasad, On simultaneous approximation by modified Lupas operators. J. Approximation Theory 45(1985), 122-128.

14. S.P. Singh, On approximation by modified Szász operators. Math. Chron. 15(1986), 39-48.

15. B.D. Vecchia, On the preservation of Lipschitz constants for some linear operators. Boll. Unione Mat. Ital., VII Ser., B-3(1989), 125-136.

16. Zhou Dingxuan, Uniform approximation by some Durrmeyer operators. Approximation Theory Appl. 6(1990), 87-100.

(Received 05.05.1993)

Author's Address:

Institute of Mathematics

Adam Mickiewicz University

Matejki 48/49

60-769 Poznań, Poland 